\title{
The Development of a Robotic Endoscope
}

\author{
A. Brett Slatkin (brett@robby.caltech.edu) Joel Burdick (jwb@robby.caltech.edu) \\ Dept. of Mechanical Engineering, CALTECH, Mail Code 104-44, Pasadena, CA 91125 \\ Warren Grundfest, M.D. \\ Cedars-Sinai Medical Center, Los Angeles, CA
}

\begin{abstract}
This paper describes the development of a prototype robotic endoscope for gastrointestinal diagnosis and therapy. The goal of this device is to access, in a minimally invasive fashion, the portions of the small intestine that cannot be accessed by conventional endoscopes. This paper describes the macroscopic design and function of the device, and the results of preliminary experiments that validate the concept.
\end{abstract}

\section{Introduction and Motivation}

This paper describes initial progress in the development of a gastrointestinal robot or robotic endoscope for traversing the human gastrointestinal system. Such a device could provide minimally invasive access to large sections of the gastrointestinal system which are currently accessible only through invasive methods.

Conventional endoscopes are highly flexible devices for minimally invasive ${ }^{1}$ inspection in interior lumens and cavities, such as the stomach, colon, urinary tract, respiratory tract, etc. Current flexible endoscopes are comprised mainly of fiber optic bundles, or distal CCD chip cameras, for transmitting an optical image. Their fiber optic bundles can also transmit laser beams that cut, cauterize, or vaporize tissue. Typically, larger diameter endoscopes also contain devices for steering their distal tips, and many permit the deployment of instruments for the surgical manipulation and dissection of tissue; such tools are often useful in the minimally invasive procurement of tissue samples.

Gastrointestinal endoscopy represents the diagnosis and treatment of alimentary canal diseases by the use of flexible endoscopes. Gastroscopes are used to visualize the

\footnotetext{
${ }^{1}$ Minimally invasive medical techniques are aimed at reducing the amount of extraneous tissue which must be damaged during diagnostic or therapeutic procedures, thereby reducing patient recovery time, discomfort, and deleterious side effects. Arthroscopic knee surgery is one widely known example.
}

inner surfaces of the stomach and colonoscopes provide visualization of the large intestine, or colon. But these two ends of the alimentary canal represent only thirty percent of the length of the gastrointestinal tract. The remaining seventy percent of the gastrointestinal tract, also known as the small intestine, cannot be reached using existing endoscopic technology. The inaccessibility of most of the small intestine arises because commercial endoscopes, while highly flexible, have only limited ability to bend at their tips and lack any means to actively control their shape along the rest of their length. Thus, endoscopic maneuverability is limited, and endoscopic surgeons must exercise substantial skill in order to position such devices deep in the body.

The latest estimates from the Journal of Clinical Gastroenterology (1992) indicate that small bowel diseases, such as Crohn's disease, ulcerative colitis, and intestinal blockage, affict $\sim 430,000$ people in the United States. Because of limitations of current endoscopic technology, the diagnosis of small intestine diseases is done either by assessing the "external" symptoms, often with radiological substantiation (e.g., via barium ingestion), or by invasive surgical exploration. And, in many instances, the current therapeutic intervention of these disease processes requires very invasive surgery, typically with a prolonged recovery period.

This paper reports our ongoing efforts to develop a robotic endoscope that can directly access and visualize, in a minimally invasive manner, the entire gastrointestinal tract. The long term goals of these efforts are to produce a surgeon guided robot that can semiautonomously locomote in the small bowel to perform medical diagnostic procedures. Such a device will eliminate or minimize the need for highly invasive diagnosis. A longer term goal is to supply therapeutic intervention when possible. For example, the robot may assist in the removal of blockages, or to accurately deliver drugs which may help in the non-invasive treatment of Crohn's disease. The required first step is to develop a machine that can dependably travel through the small intestine. Unless 
this technical challenge is overcome, none of the other medical goals can be reached. The focus of this paper is on the robotic locomotion mechanism.

\section{Relation to Previous Work}

Endoscopy is generally considered to be a mature medical technology with many commercial devices available for the diagnosis and treatment of disease processes found within various lumens of the human body. To use existing endoscopes, the endoscopist holds the proximal end and advances the distal end by pushing from outside of the body. It is desired that the endoscope slide easily inside of the tunnel-like lumen, but often this is not the case. Because many lumens in human physiology are curved along their length, endoscopes designed to traverse them must be sufficiently flexible with respect to lateral bending in order to follow the contours of the lumen. Unfortunately, these devices are advanced in the lumen by pushing from behind, which requires them to be sufficiently stiff to prevent buckling. Since the lumen can laterally support the endoscope along its length, one might believe that buckling would not be a problem. However, this is a misconception for two reasons. Firstly, biological lumens can be extremely flexible (more so than the endoscope itself), and, thus, they do not provide substantial support against buckling. And secondly, the distal end of the endoscope can "snag" on undulations in the internal surface of the lumen producing rather than preventing buckling during endoscope advancement. Buckling is a main cause of patient discomfort and is potentially injurious to fragile, diseased, surrounding tissues. Moreover, this buckling phenomenon also limits the depth of penetration as it is more likely to occur when the unsupported length of the endoscope increases.

Many have recognized that improvements in endoscopy could be effected by introducing actively controlled electromechanical articulation along the endoscope's length. For example, Sturges et. al. [14] have investigated the use of articulated bead-chain mechanisms for endoscope design. Fukuda and coworkers have likewise engineered prototype catheters which can actively bend along their length [2]. Ikuta and collaborators have developed hyper-redundant robotic endoscope prototypes $[9,8]$. But in all of these cases the devices are advanced into the body by forces produced at their proximal ends, which are located outside of the patient. This type of actively articulated endoscope design inherently limits its overall length and, hence, its ultimate reach into the body.

In a more general context, it has recently been rec- ognized that robotic technology has the potential for significant applications in medicine. Robotic technology has already been applied to the realm of invasive surgery. For example, robots have successfully been used to assist hip replacement surgery [10] and cranial surgery $[12,11]$. However, less progress has been made in the use of robotic technology for minimally invasive medical practice. Recently, robots have been developed to assist in laparoscopic surgery ${ }^{2}[15,4]$; however, these developments are aimed at improving surgical procedures which are already performed in a minimally invasive fashion. It is the opinion of the authors that robotic technology may have a greater impact in providing minimally invasive, alternative surgical methods to currently invasive practice.

This shift to minimally invasive medical surgery is one of the biggest trends in medical practice in the 1990's [5]. Arthroscopic knee surgery, colonoscopic polypectomy, and laparoscopic gall bladder removal are widely recognized examples of this trend. Minimally invasive surgery typically involves the use of slender surgical instruments inserted into body cavities through naturally occuring or surgically produced orifices. Such techniques can vastly reduce the amount of peripheral cutting required to reach the site of diseased or injured tissue. This can translate to dramatic improvements in patient care at greatly diminished health care costs. These improvements include, but are not limited to, reduced patient recovery times and reduced postoperative discomfort. There can be tremendous fiscal incentives as well for the adoption of these techniques. Approximately 21,000,000 surgeries are performed each year in the United States [5]. It is estimated that $8,000,000$ of these surgeries can potentially be performed in a minimally invasive manner; however, only about $1,000,000$ surgeries are currently so performed annually due in part to limitations in minimally invasive surgical technology. The complete adoption of minimally invasive techniques could be expected to save up to $\sim \$ 28,000,000,000$ annually in hospital residency costs and lost patient wages [5].

One of the biggest technological impediments to increasing usage of minimally invasive approaches is lack of minimally invasive access to interior body cavities. While the focus of our current development effort is a device for minimally invasive access to the gastrointestinal system, we hope that successful deployment of this robot will pave the way for other applications of robotic technology to minimally invasive medicine.

As described below, our design is reminiscent of "pipe

\footnotetext{
${ }^{2}$ Laparoscopes are rigid optical tubes which are used for minimally-invasive inspection and surgery inside the abdominal cavity.
} 
crawling" robots that have previously been investigated for inspection of buried pipes and channels. Fukuda and coworkers have developed self-propelled robotic systems for inspecting small and medium size pipes[3], and Shishido et. al. [13] have been granted a United States patent for such an invention [13]. While the topology of the human intestine is analogous to a pipe, there are many significant differences which prevent a simple adaptation of prior pipe crawling robot principles to our problem. First of all, the diameter of the human intestine can vary by a factor of four over its length. Conventional pipe crawling designs do not handle such variations. And, in addition, the intestine is highly flexible, fragile, and slippery. Thus, the traction mechanisms used in many prior pipe crawling devices would likely cause significant injury to the intestinal lining (provided they could produce sufficient traction at all).

It was with consideration of these concerns that the authors have conceived and patented the robotic endoscope described herein $[7,6]$.

\section{The Robot Endoscope}

The following sections of this paper describe a class of mechanisms and locomotion modalities which may be incorporated into a robotic endoscope that can propel itself in a flexible lumen for the purposes of medical diagnosis and therapeutic intervention. After providing a detailed presentation of the locomotion concepts for endoscopic propulsion, this section will describe the mechanical components and electronic systems that implement these concepts in our prototype.

\subsection{System Overview}

Figure 1 shows a schematic overview diagram of the prototype endoscopic system, while Figure 2 shows a photograph of one of the prototypes that have been developed to date. There is an endoscopic robot with a trailing cable which consists of: electrical wiring for control signals; tubing to connect the pneumatic actuators to high and low pressure sources; and an optical fiber bundle for illumination and imaging of the area in front of the robot. The optical fiber bundle consists of a 1.4 millimeter diameter flexible endoscope inserted through a channel placed within the robot. The electric wiring carries currents to the solenoid valves located within the robot. These currents are sourced by interface electronics which interpret signals from an external computer. Fluid power actuation for these devices was chosen because conventional endoscopic procedures require carbon dioxide gas, saline solution, and partial vacuum for

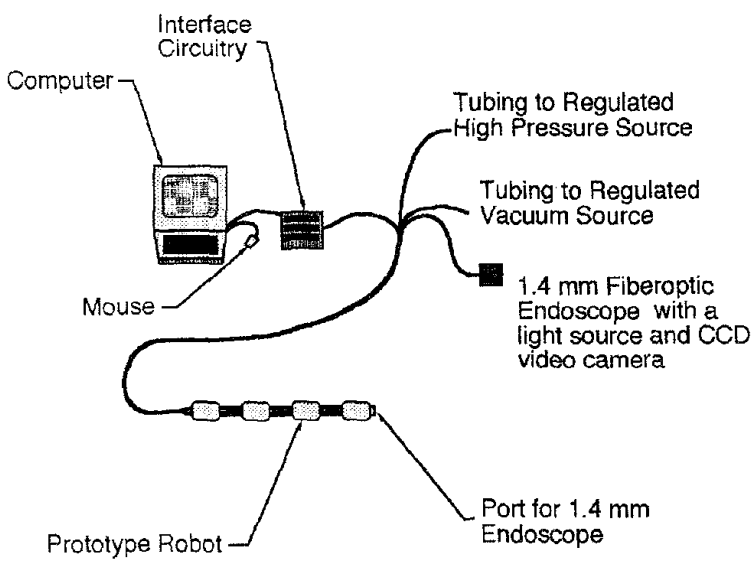

Figure 1. Schematic Overview of the Robotic Endoscope System

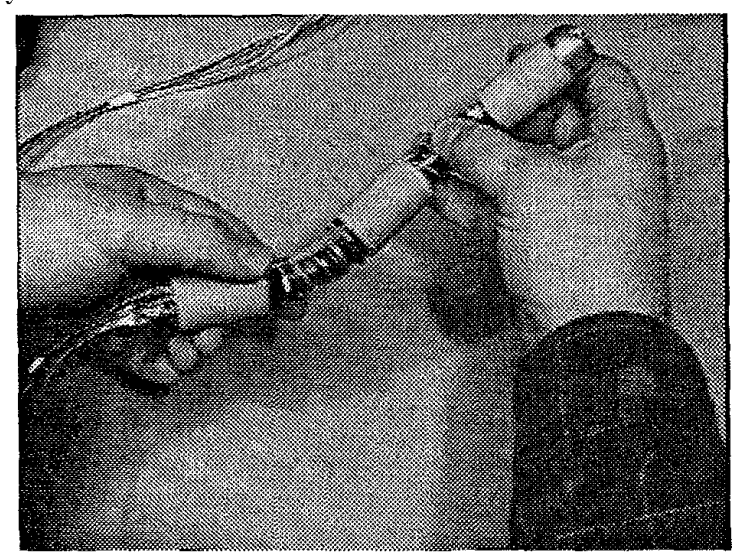

Figure 2. Photograph of one Robotic Endoscope Prototype (a 3 gripper $/ 2$ extensor configuration)

insufflation, irrigation, and suction of gastrointestinal lumens. Hence, it is convenient and efficient to use these fluids as power sources for locomotion. Additionally, since large amounts of pneumatic or hydraulic mechanical power can be controlled by small electric signals, this approach minimizes the danger of electric shock to the patient. Another safety measure of these designs is that the working pressures of the actuators are kept intentionally small. In our prototypes, the high pressure source is typically maintained at $12 \mathrm{psig}$, while the low pressure is nominally $-14.2 \mathrm{psig}$ (vacuum).

Referring to Figure 3, our endoscopes appear outwardly to be electromechanical analogs of segmented worms, such as earthworms. In order to propel itself, this robotic endoscope employs mechanisms along its length which can be described as "grippers" and "extensors." The primary purpose of the grippers, or traction devices, is to provide traction against the lumen wall by expanding radially outward. The extensors provide exten- 


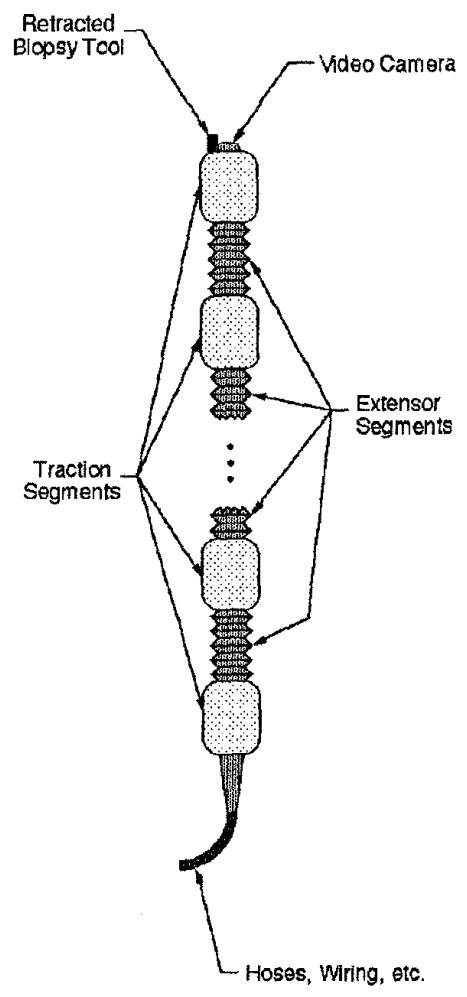

Figure 3. Schematic Diagram of the Robotic Endoscope

sibility between the grippers-i.e., they cause the mechanism to locally expand or contract in length. Locomotion is the process of generating net displacement of the robotic endoscope inside a flexible lumen by specific sequences of gripping and stretching actions. Such sequences are commanded by an external computer, and, thus, changes in the selection and control of mechanical movements can be easily accomplished in software. Furthermore, these machines are not limited by their mechanical design to locomote by a particular gripper and extensor sequence. Practical experience dictates that robust locomotion of the machine through lumens which exhibit changing geometric or material characteristics along their length (e.g. varying cross-sectional diameter) require repeated changes in gaits, or maneuvering sequences.

\subsection{Methods for Locomotion}

A gait is a distinct cycle of changes in the state of the component gripper and extensor segments that leads to a unit of displacement, which we term a stride. The length of the displacement is termed the stride length. Repetition of a gait leads to net displacement, or locomotion. Depending upon the number and arrangement of traction and extension mechanisms, a given device will typically be able to implement more than one gait.

In Figures 4 through 8 , several gaits are shown for en-

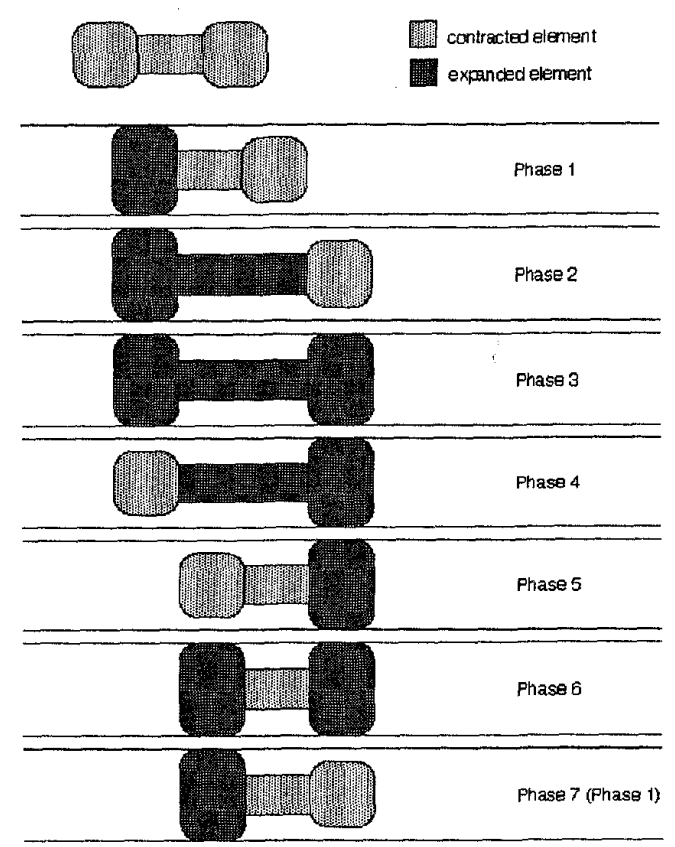

Figure 4. Schematic Diagram of Locomotion for a 2 Gripper, 1 Extensor Mechanism

doscopes that are comprised of two, three and five gripper segments. In these figures, the phase, or state, of the machine is shown for sequential moments of time. Between the given phases, the states of expansion of individual gripper and extensor segments change. Since subsequent phases of the locomotion gaits are portrayed in these figures as vertically displaced lumens, the stride length of each gait shown can be deduced. While the lumen is represented as a straight tube, the inch-worm type locomotion schemes will work if the lumen is curved and has reasonable variations in its cross-sectional shape and diameter. This is possible because the extensor devices are engineered to exhibit a useful form of passive compliance to lateral forces. And active control of lateral bending of the extensor segments is not precluded (and may, in fact, be desired).

\subsection{The Gait of a 2-Gripper/1-Extensor Mechanism}

For the purposes of illustration, let us first consider a locomotion method for a device consisting of two traction mechanisms and one extensor mechanism, as depicted in Figure 4. This is the simplest design that can generate endoscopic locomotion.

A 2-gripper/1-extensor mechanism can implement only one inch-worm type gait. The sequencing of the gait for forward motion proceeds as follows (in the diagram, forward motion means motion to the right). Let us assume that the sequence begins with the aft traction device 
expanded to grip the lumen wall (as indicated in the part of the diagram labeled "Phase 1"). Meanwhile, the forward gripper and the extensor are in their retracted states. The extensor device is then extended (Phase 2 ). While the extensor will typically be expanded to its full length, partial extension is possible. If the lumen is curved, the lateral compliance (active or passive) of the extensor will cause the expanding extensor to move in the principal direction of the lumen. After the extension is complete, the forward traction device is expanded to grip the lumen wall (Phase 3 ). After the forward gripper has extended, the rear gripper is retracted (Phase 4). Next, the extensor is retracted (Phase 5). Here again, the extensor will typically be retracted to its shortest position, though partial retraction is possible. Subsequently, the rearward gripper is expanded to grip the lumen wall (Phase 6). Finally, the forward gripper is retracted. At this point, the device is in the same state as the beginning of this sequence. However, the robot has moved forward by a single stride length. Assuming no slip, the stride length is the difference between the extended extensor length of Phase 2, and the retracted extensor length of Phase 5 .

These steps comprise a single gait cycle. This cycle can be repeated to provide continual motion. This cycle can also be reversed to implement motion in the rearward, or opposite, direction. Loosely speaking, this gate may be termed a "standing wave" gait (using the terminology of [1]), since the movement of the extensor can be loosely characterized as a body fixed oscillation.

\subsection{Some Gaits for a 3-Gripper/2-Extensor Mechanism}

Let us now consider means by which a device consisting of 3 grippers and 2 extensors (like the robot of Figure 2 ) can locomote. This version can effect at least ten distinct gaits. We discuss here only two of these gaits in detail to point out some important characteristics of our device. Self-explanatory schematics of some of the other gaits are also included in Figures 7 .

The first gait for this robot that we will characterize is elucidated in Figure 5 . In Phase 1, all of the grippers and extensors are in their expanded states. The rear gripper is retracted in Phase 2. Next, the rear extensor is retracted (either partially, or fully) in Phase 3. Subsequently, the rear gripper is expanded to make contact with the lumen (Phase 4). In Phase 5, the middle gripper is retracted. Next, the forward extensor is retracted while the rear extensor is expanded (Phase 6 ). Then, the middle gripper is expanded to make contact with the lumen. In Phases 8 and 9, the forward gripper is retracted, and then the forward extensor is

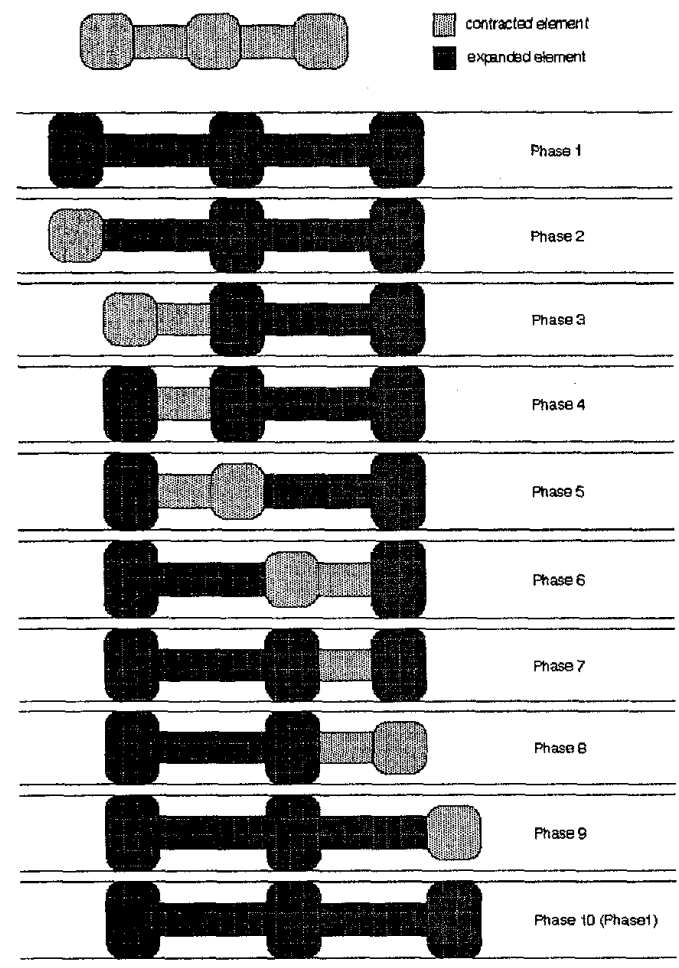

Figure 5. Schematic Diagram of a Wave-like Locomotion Gait for a 3 Gripper, 2 Extensor Mechanism

extended. Finally, in Phase 10, the forward gripper is again expanded. At this point the mechanism has returned to its original state, but moved forward by one stride length. This cycle can be repeated for continual forward movement, or it can be reversed for rearward motion.

From the explanation of this gait sequence, it can be seen that contact points with the lumen advance along the lumen wall in a "wave-like" pattern. Hence, we can describe this motion sequence as a "traveling wave gait" in much the same manner as those described in [1]. It should be recounted that lateral compliance of the extensors will enable the mechanism to move in curved lumens as well. Furthermore, it is clear that at any instant, only relatively short segments of the device are being pushed forward through the lumen. This overcomes the buckling problems inherent in conventional endoscope designs.

It should also be noted that in this gait, at least 2 gripping devices make contact with the lumen at all times. Consequently, a three gripper device employing this gait would be able to more robustly grasp the lumen than the 2 gripper design of Figure 4.

Figure 6 shows a second gait example for a 3 -gripper/2extensor device. It should be noted that in all phases 


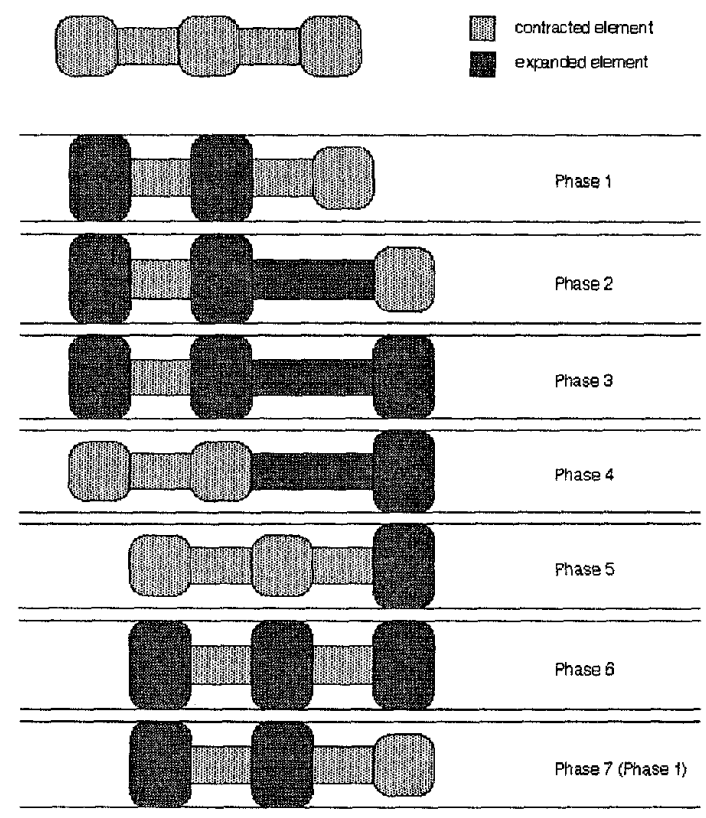

Figure 6. Schematic Diagram of Locomotion for a 3 Gripper, 2 Extensor Mechanism
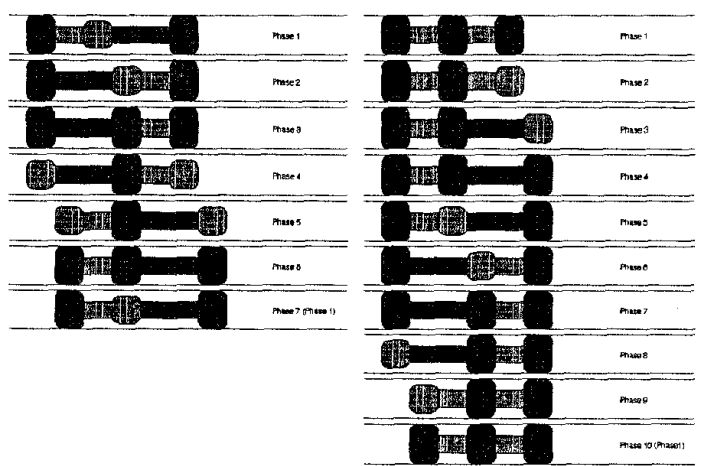

Figure 7. Schematic Diagrams of Additional Locomotion Gaits for a 3 Gripper, 2 Extensor Mechanism

of this gate, the rear extensor is retracted. The same exact sequence of maneuvers could also be used if the rear extensor were extended. In other words, this gate makes no use of the rear extensor. Thus, if the rear extensor were to become inoperable during use, this gait could be used to enable endoscope locomotion even in the event of the failure of the rear extensor. Similarly, a gait exists which will be robust to a failure of the front extensor. And, thus, because the endoscope can switch between gaits, this design is robust to a single extensor failure. In addition, one can also derive a gait which will be robust to the failure of one of the grippers (assuming that the gripper fails in its retracted state). Overall, the 3 gripper $/ 2$ extensor device can exhibit ten substantially different gaits.

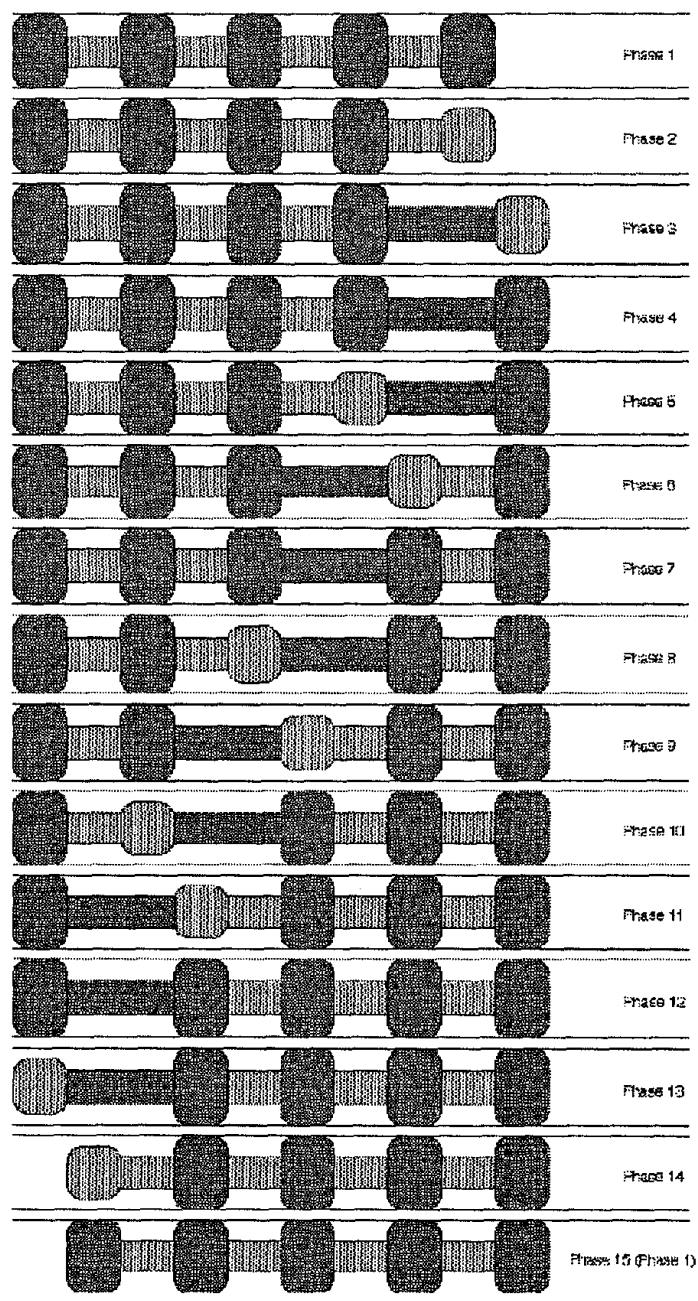

Figure 8. Schematic Diagram of Wave-like Locomotion for a 5 Gripper, 4 Extensor Mechanism

\subsection{Gaits for Designs with Numerous Grip- pers and Extensors}

As the number of grippers and extensors in the robotic endoscope design increases, the number of possible gaits increases as well. For example, Figure 8 shows one possible gait for a device consisting of 5 grippers and 4 extensors. Clearly, one cannot list all possible gaits for all possible combinations of extensors and grippers. But for a given mechanism, it should be evident that one could systematically develop the sequencing of all possible gaits using the ideas outlined above.

In general, it is advantageous for an endoscope implementation to be capable of a large number of gaits. This in turn implies that highly adaptable endoscopes will have many gripper and extensor segments. As shown above, some gaits typically have more grippers in contact with the lumen. These gaits tend to be more sta- 
ble, though the progress of the device through the lumen tends to be slower. A slower but more robust gait would be useful when the robot moves from a region of the lumen which produces strong traction into one which does not. Further, gaits with more grippers in contact can in some instances generate greater forward forces, which might be useful for unblocking intestinal blockages. Conversely, it may be desirable to select a faster gait when the robot moves into a region of strong traction from one of weaker traction. In addition, there exist gaits which are robust to the failure of particular components, as illustrated in Figure 6 . The ability to switch between gaits as the situation dictates is a key feature of this device.

\subsection{Component Designs}

This section describes the mechanisms which implement the actions described above.

Traction Segment Designs. The action of a gripper or traction segment is to grasp the inside surface of the encompassing lumen by locally increasing its effective diameter. It is important to note that these mechanisms require special features in order to guarantee that this medical robot is safe and efficacious. With regard to the gripper segments, their design should allow the machine to gently grasp the inner linings of the lumen so as to minimize any potential injury to the possibly fragile tissues. Although many different mechanisms can be conceived which will produce this effect, ones based on fluid power actuation were chosen for our early prototypes. The contracted diameter of the gripper segments of the robot shown in Figure 2 is seven-eighths of an inch; also, these segments are 1.5 inches in length.

In each of the prototypes built to date, the gripping action of the traction segments has been accomplished by inflation of toroidal balloons located around their periphery. A schematic of the most recent prototype gripper segment is shown in Figure 9. It consists of two, two-way, normally closed solenoid valves for inflation and deflation of the traction balloon. The inflation valve, $\mathrm{C}$, is opened for a specified duration until the balloon, A, is sufficiently distended by fluid from the high pressure source line, $\mathrm{E}$; this valve is then closed. This design allows the segment to maintain traction without the continuous dissipation of power. When traction is no longer required, the deflation valve, $D$, is opened which vents the balloon to a partial vacuum line, $F$, for a given length of time. This valve is then closed; thus, the traction segment can also remain in its contracted state without continuous expenditure of energy.

Extensor Segment Designs. The extensors pro-

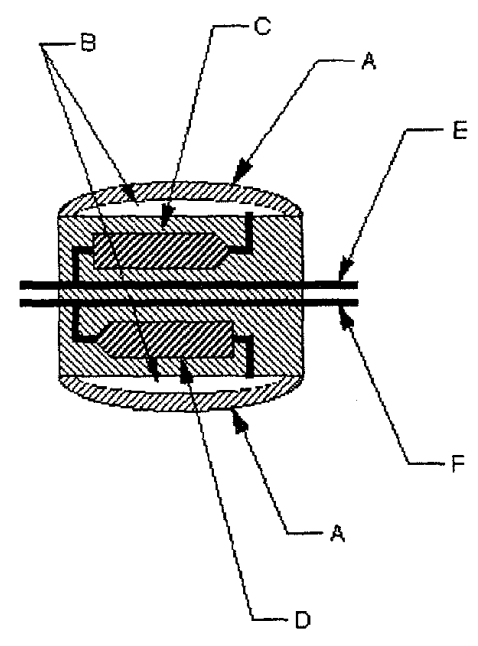

Figure 9. Schematic Section View of a Traction Segment

vide the local axial expansion and contraction required for inch-worm type locomotion. Since lumens of human physiology are often substantially curved along their length, the endoscope must be able to bend laterally. Our recent prototypes employ a modified bellows to provide extension of over fifty percent of the contracted length of 1.35 inches. Internal stiffening elements provide the appropriate bending and longitudinal stiffnesses. This modified bellows structure allows the extensor segments to expand preferentially in the axial direction under internal pneumatic inflation while simultaneously acting to provide the desired lateral bending compliance. In the present prototypes, on-board solenoid valves are used to control the flow of fluid in and out of the extensor segments.

\subsection{Electronic Gait Control}

In the present design, the traction and extension actions are controlled by pneumatic valves located along the length of the machine. The valves are in turn controlled by a computer, via a custom electronic interface. This interface allows the computer to control up to sixty-four valves through an eight bit data port. This interface can control endoscopes with maximum of thirty-two fluid power actuators (as each actuator requires two valves), or in practice, up to 16 grippers and 15 extensors. As such, the authors anticipate that this interface will be useful in the control of all future prototypes of this type of endoscopic robot.

Locomotion is generated when individual gripper and extensor expansion states are changed according to particular sequences, or gaits. These states are controlled 


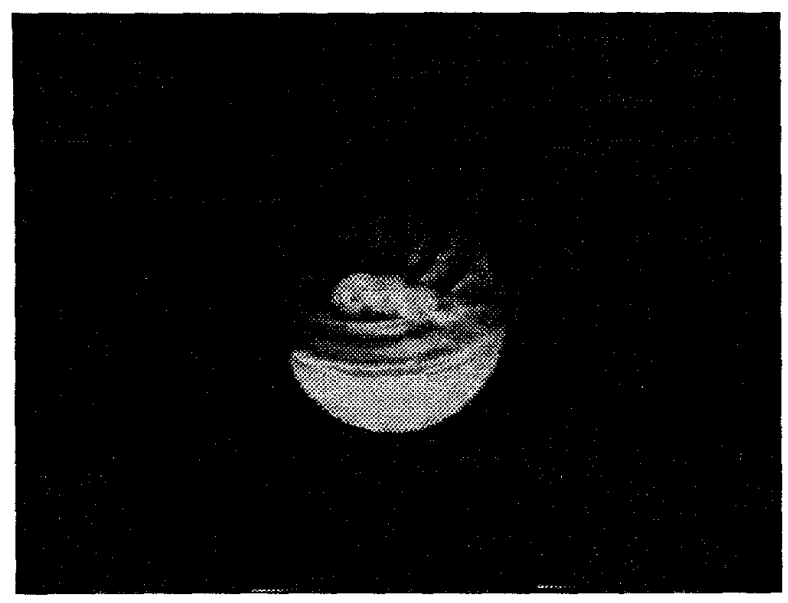

Figure 10. Approach to a Simulated Polyp

by the opening and closing of internal valves connected to the high and low pressure source lines. Each gait is described by a sequence of phases. For any change of phase within a gait, specific valves are opened and then closed. The electronic amplifier that is connected to each valve has an associated address in the digital interface circuitry. Each phase is used as an index for a lookup table which contains the valve addresses and valves states that should be modified during that phase transition. The rate at which the computer cycles through the phases determines the speed of the machine, while the order of the cycle determines the forward or backward motion. Simple keystroke commands can be used to modify pertinent locomotion parameters, such as: the selection of the locomotion gait, the direction of locomotion, the speed of locomotion, and the duration that the valves are kept open during a given change of phase (which controls the degree of pneumatic actuator inflation or deflation).

\section{Experiments and Results}

To date, we have developed several 2-gripper/1-extensor and 3-gripper/2-extensor endoscope prototypes (such as the one in Figure 2. This section describes the results of early experiments used to test the locomotion concepts and mechanical designs of these endoscopic robots. These experiments include tests of the components and systems in fairly rigid urethane tubing as well as swine intestines.

\subsection{Experiments in Urethane Tubing}

The first goal of these experiments is to verify the reliability of the locomotion and and structural integrity of these prototype devices. The intestines through which

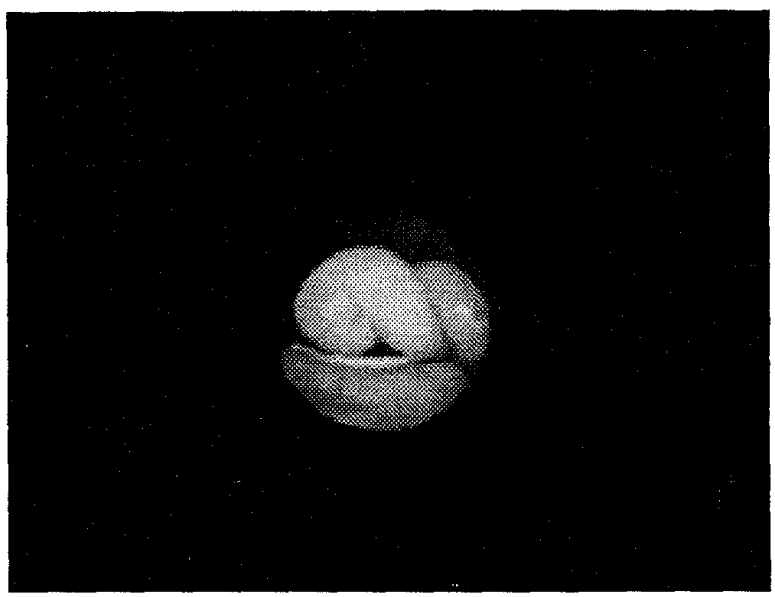

Figure 11. Approach to a Simulated Polyp

they are intended to travel are extremely flexible, slippery, and curved with relatively small radii of curvature. Although the urethane tubing is quite rigid, by lubricating its interior surface and bending it along its length, the ability of our robots to locomote in slippery and curved environments can be tested. The use of this laboratory apparatus continues to provide proof of concept for the hardware and software development of these devices. The prototype of Figure 2 incorporates a 1.4 millimeter fiberoptic endoscope for the illumination and imaging of the robot's environment. Figures 10 and 11 are images taken from within the tube as the robotic endoscope was driven under remote control to investigate a simulated polyp.

The software for this prototype can command the robot to locomote in any of eight gaiting sequences as selected by the user, including ones which provide reliable locomotion with failed actuators. This turned out to be especially convenient when one of the extensor actuators failed during one experiment. While the robot was still located within the tube, the user selected a gait which did not require use of that extensor, and, thus, the test continued successfully. The unfortunate structural failure of one component of this robot demonstrated the practicality of redundancy in the design of the machine. It is expected that subsequent versions of these robotic endoscopes will consist of many more segments than this one. This will result in a machine which can suffer multiple component failures and yet maintain its ability to complete its mission.

\section{Animal Experiments}

In addition to the "clean" testing of these robotic devices, a few animal studies have also been undertaken. It is accepted that certain animal models represent reasonable equivalents to human physiology. In particular, 


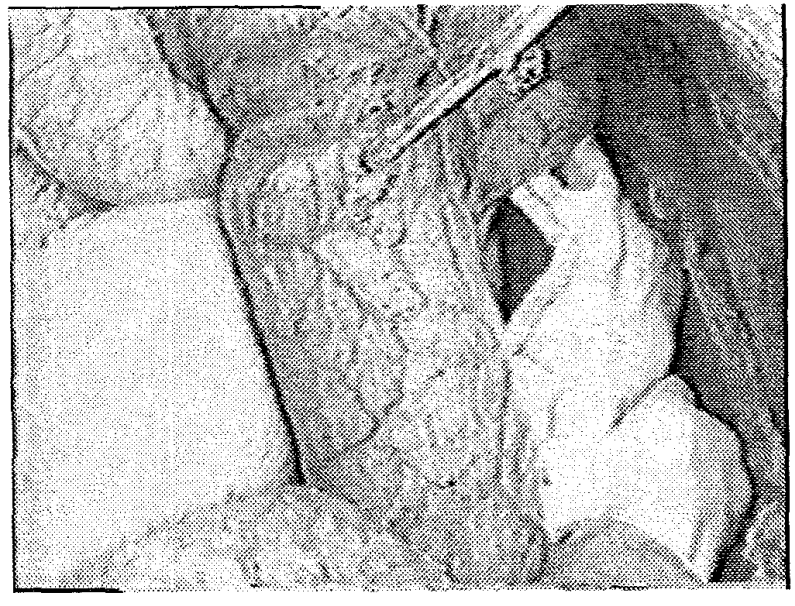

Figure 12. Contracted Traction Segment Within the Small Intestine of a Pig

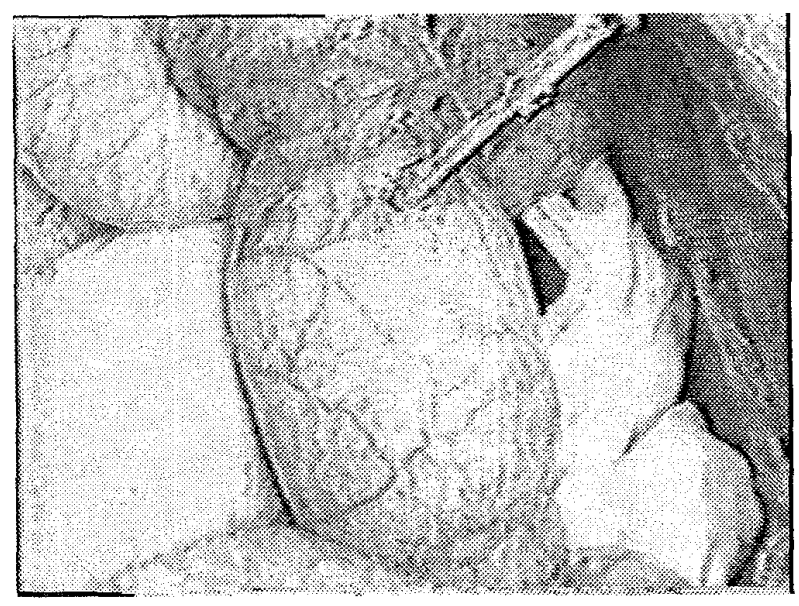

Figure 13. Expanded Traction Segment Within the Small Intestine of a Pig

the intestines of an adult pig strongly resemble those of a juvenile human in size and mechanical properties. And the intestines of a juvenile human are typically smaller and considerably more flexible than those of an adult human. As such, the intestines of a pig are a conservative model with which to test an actively propelled endoscopic robot since it is considered to be more difficult to move through a smaller and more flexible lumen than through a larger, rigid one.

These in vivo experiments took place in a surgical laboratory with the animal under general anesthesia as was required to keep the appropriate tissue alive. The first in vivo experiment was a simple dissection of intestinal tissue for analysis of its mechanical properties. The results of this test indicated that the development of

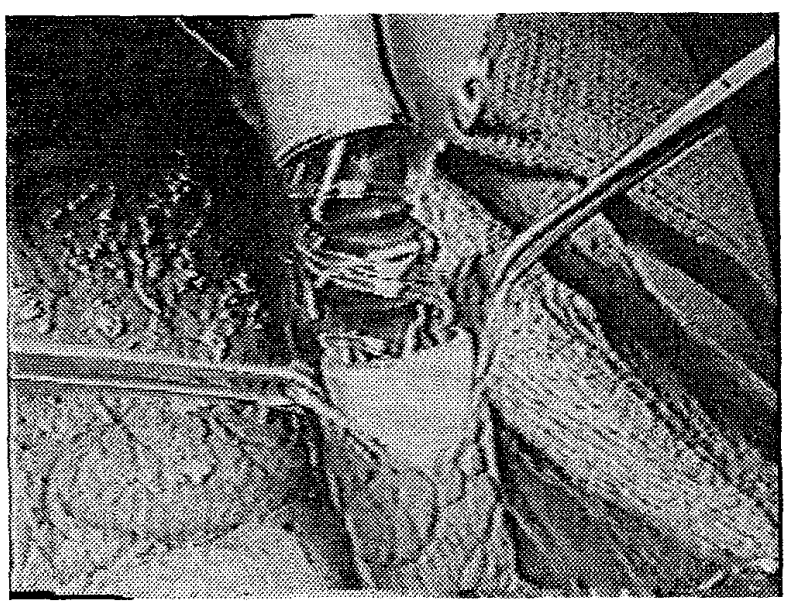

Figure 14. Inserting a Prototype Robot into the Small Intestine of a Pig

a machine which could locomote within this environment would likely be quite difficult; the flexibility and lubricity of this lumen would probably prove to be problematic. However, a subsequent experiment to test the available traction of a balloon type gripper device indicated that very substantial traction was indeed possible, and this result was encouraging. Figures 12 and 13 show a single traction segment undergoing expansion within the small intestine of a pig. At this point, it was decided that an attempt should be made to achieve locomotion in vivo with an existing prototype. Figure 14 shows a prototype robot as it was inserted through an incision into the small intestine of a pig. Ultimately, these devices will be launched from an endoscopic delivery system from either the stomach or the colon, but, to date, they have been introduced into the small intestines through surgical incisions. Since the outer surface of the small intestines has been visible during these experiments, it was possible to observe the movements of the prototype inside the lumen. Although this machine could indeed move through a portion of the small intestine, it was clear that further development is required to support extensive in vivo experiments.

\section{Conclusions and Future Work}

There is a clear need for endoscopic devices that can access the human small bowel in a minimally invasive fashion. In this paper we described our preliminary efforts to develop such a system. Our experiments have shown that the locomotion concepts work extremely well for machines moving within relatively rigid tubes and appear to apply to the flexible and slippery small intestines as well. These efforts represent an encouraging first step toward robotic endoscopy.

Our ongoing work is focused on the design and fabrica- 
tion of a new modular endoscope robot for continuing in vivo locomotion experiments. Our short term goal is to reduce the robot's as much as is practical. To achieve this reduction in size, we are currently developing a new generation of miniature pneumatic valves, since the size of the on-board valves represents the limiting constraint on overall endoscope size. The newest valves are roughly sixty percent of the size of those used in the previous prototypes, and should allow us to produce a robot with a $14 \mathrm{~mm}$ diameter.

In Section 4.1, it was noted that the urethane tubing experimental apparatus cannot precisely match the environment within the intestines due to its structural rigidity. But, within the body, there exist other physiological lumens which are considerably stiffer and, therefore, would be well modelled by the urethane tubing. Unfortunately, these tubes in the body are significantly smaller in diameter than the intestines, and, thus, an endoscopic robot designed to traverse them must likewise be considerably smaller than those intended for the intestines. Therefore, it is a future goal of these researchers to build yet smaller versions for these applications.

\section{References}

[1] G.S. Chirikjian and J.W. Burdick. The kinematics of hyper-redundant locomotion. IEEE Trans. on Robotics and Automation, to appear, 1994.

[2] T. Fukuda, S. Guo, K. Kosuge, F. Arai, M. Negoro, and K. Nakabayashi. Micro active catheter system with multi degrees of freedom. In Proc. IEEE Int. Conf. on Robotics and Automation, San Diego, May 1994.

[3] T. Fukuda, H. Hosokai, and M. Uemura. Rubber gas actuator driven by hydrogen storage alloy for in-pipe inspection mobile robot with flexible structure. In Proc. IEEE Int. Conf. on Robotics and Automation, pages 1847-1852, Scottsdale, AZ, 1989.

[4] J. Funda, R. Taylor, S. Gomory, B. Eldridge, K. Gruben, and M. Talamini. An experimental user interface for an interactive surgical robot. In Proc. First Int. Symp. on Medical Robotics and Computer Assisted Surgery, Pittsburg, PA, 1994.

[5] The Wilkerson Group. New developments in medical technology. 1991.

[6] W.S. Grundfest, J.W. Burdick, and A.B. Slatkin. Robotic endoscopy. U.S. Patent pending.
[7] W.S. Grundfest, J.W. Burdick, and A.B. Slatkin. Robotic endoscopy. U.S. Patent No. 5337732, August 161994.

[8] K. Ikuta, M. Nokata, and S. Aritomi. Hyperredundant active endoscope for minimum invasive surgery. In Proc. First Int. Symp. on Medical Robotics and Computer Assisted Surgery, Pittsburg, PA, 1994.

[9] K. Ikuta, M. Tsukamoto, and S. Hirose. Shape memory alloy servo acuator system with electric resistance feedback and application for active endoscope. In Proc. IEEE Int. Conf. on Robotics and Automation, pages 427-430, Tokyo, 1988.

[10] B. D. Mittelstadt, P.H. Kazanzides, J. Zuhars, B.Williamson, P. Cain, F. Smith, and W.L. Bargar. The evolution of a surgical robot from prototype to human clinical use. In Proc. First Int. Symp. on Medical Robotics and Computer Assisted Surgery, Pittsburg, PA, 1994.

[11] R. Rohling, P. Munger, J.M. Hollerbach, and T. Peters. Comparison of relative accuracy between a mechanical and an optical position tracker for image-guided neurosurgery. In Proc. First Int. Symp. on Medical Robotics and Computer Assisted Surgery, Pittsburg, PA, 1994.

[12] H.J. Schulz, T. Lutze, and H.P. Trummler. Requirements of a planning and navigation device used in neuro-, ent-, and maxillocraniofacial surgery. In Proc. First Int. Symp. on Medical Robotics and Computer Assisted Surgery, Pittsburg, PA, 1994.

[13] Y. Shishido, H. Adachi, H. Hibino, T. Yamamoto H. Miyanaga, S. Takayama, Y. Ueda, Y. Aoki, and S. Yamaguchi. Pipe-inspecting apparatus having a self propelled unit. U.S. Patent No. 5090259, March 251986.

[14] R.H. Sturges and S. Laowattana. A flexible, tendon-controlled device for endoscopy. In Proc. IEEE Int. Conf. on Robotics and Automation, Sacramento, CA, 1991.

[15] D.R. Uecker, C.L. Lee, Y.F. Wang, and Y. Wang. A speech-directed multi-modal man-machine interface for robotically enhanced surgery. In Proc. First Int. Symp. on Medical Robotics and Computer Assisted Surgery, Pittsburg, PA, 1994. 